\title{
Akzeptanz \\ von Technikeinsatz \\ in der Pflege
}

Zwischenergebnisse einer Befragung unter professionell Pflegenden

Jan C. Zöllick, Adelheid Kuhlmey, Ralf Suhr, Simon Eggert, Johanna Nordheim und Stefan Blüher

17.1 Einführung: Technikeinsatz, Digitalisierung und Automatisierung in der Pflege - 212

17.2 Forschung zu technischen Assistenzsystemen im Gesundheitsbereich - 213

17.3 Befragung zur Akzeptanz von Technikeinsatz in der Pflege - 214

$17.4 \quad$ Fazit -216

$$
\text { Literatur - } 217
$$




\section{- Zusammenfassung}

Der Einsatz von Technik in der Pflege wird im Spannungsfeld von zunehmendem Pflegebedarf einerseits und fehlenden Fachkräften andererseits diskutiert. Eine Befragung von professionellen Pflegekräften $(n=127)$ ergab, dass technische Assistenzsysteme zur körperlichen Unterstützung der Pflegearbeit geschätzt und akzeptiert sind; solche, die soziale und emotionale Zuwendung bieten sollen, jedoch als weniger hilfreich, entlastend oder nützlich gesehen werden. Ein solcher Technikeinsatz führe laut den Befragten eher zum Verlust menschlicher Wärme und stehe im Kontrast zum beruflichen Selbstbild der Pflege. Ein auffälliges Forschungsergebnis ist, dass die fehlende Bereitstellung von Technik am Arbeitsplatz als ein wesentliches Hemmnis für ihre Nutzung in der Pflege gesehen wird. Wenn Technik vorhanden ist, wird sie hingegen von den meisten der befragten professionellen Pflegekräfte genutzt.

Many see technology in care as a remedy for tensions between an increasingly ageing population that is in need of care and the shortage of skilled personnel to fulfill these tasks. A survey of professional nurses $(n=127)$ suggests that technologies for physical support are appreciated and accepted, whereas technologies for social and emotional support are seen as less helpful, exonerating, and useful. This second application of technology would lead to the loss of interpersonal warmth and stands in contrast to the professional ethics of care. A bottleneck preventing wide-ranging use of technology in care seems to be their missing provision at the workplace. When technology is provided, most nursing staff uses it regularly.

\subsection{Einführung: Technikeinsatz, Digitalisierung und Automatisierung in der Pflege}

Der Einsatz von Technik in der Pflege ist ein hochaktuelles Thema, das einerseits Potenziale für die Entlastung von Pflegenden, Aufrecht- erhaltung der Selbständigkeit sowie Schutz der Würde von (potenziell) Pflegebedürftigen aufzeigt. Andererseits weckt es Bedenken hinsichtlich des Berufsbildes der Pflege, der Arbeitsplatzsicherheit von Pflegekräften sowie der sozialen und emotionalen Unterstützung der Pflegebedürftigen.

Im Pflege-Report 2017 systematisierte Uwe Fachinger technische Assistenzsysteme anhand ihrer Interaktion und Vernetzung in drei Generationen (Fachinger 2017). Die erste Generation beinhaltet etablierte Systeme zur Pflegeunterstützung ohne Informationsaustausch, bspw. Hebehilfen. Zur zweiten Generation zählt Fachinger (2017) solche mit Informationsaustausch, bspw. Telemedizinsysteme oder Serviceroboter. Die dritte Generation umfasst vernetzte Systeme, die (vermeintlich) eigenständig Aufgaben erledigen, bspw. soziale Roboter zur Interaktion mit pflegebedürftigen Menschen oder eigenständig (re-)agierende Wohnsysteme.

Seit dem Erscheinen des Pflege-Reports 2017 hat besonders die dritte Generation an Pflegeassistenzsystemen größere Aufmerksamkeit erhalten. So initiierte beispielsweise das Bundesministerium für Bildung und Forschung das Programm „Bedarfsgerechte Pflege", wodurch Deutschland zum Leitanbieter für Pflegetechnologien und Medizintechnik avancieren solle (BMBF 2018). Zudem legten im deutschsprachigen Raum gleich zwei wichtige politische Akteure Berichte zu Robotik in der Pflege vor. Die Bioethikkommission beim österreichischen Bundeskanzleramt sieht die Bedürfnisse alter Menschen als zentral und ihre Privatsphäre als besonders schützenswert in dieser sehr intimen Interaktion mit der neuen Technik (Bioethikkommission 2018). Der Ausschuss für Bildung, Forschung und Technikfolgenabschätzung des deutschen Bundestages (Rossmann et al. 2018) diskutiert Roboter zur physischen und sozialen Unterstützung als mögliche Lösungsansätze des demografiebedingt steigenden Pflegebedarfs bei gleichzeitigem Fachkräftemangel. Ganzheitliche technische Systeme hätten jedoch momentan nur in geringem Ausmaß Marktreife erlangt. Daher 
besteht für den Ausschuss ,,in Bezug auf regulative Fragen der Technikanwendung derzeit kein vordringlicher Handlungsbedarf“ (Rossmann et al. 2018, S. 24). Demgegenüber zeichnet die Stiftung Münch in ihrer Publikation „Robotik in der Gesundheitswirtschaft" ein deutlich optimistischeres Bild (Klein et al. 2018). Dort finden sich Beispiele zahlreicher existierender Systeme in 16 Anwendungsfeldern und Ergebnisse von 17 Experteninterviews über die $\mathrm{Zu}$ kunft von Technik in der Pflege. Nahezu alle diskutierten Szenarien mit Zeithorizonten bis 2020 oder 2030 - bspw. Exoskelette, emotional und sozial agierende Roboter für therapeutische Zwecke oder Reinigungs- und Desinfektionsroboter - wurden von den Expertinnen und Experten inhaltlich positiv bewertet. Trotz vereinzelter Skepsis zu den Einsatzszenarien und Zeithorizonten wird davon ausgegangen, dass der Einsatz von Technik der dritten Generation in der Pflege schon in naher Zukunft deutlich zunehmen wird.

Die Bevölkerung steht dieser Entwicklung tendenziell zustimmend gegenüber, wie aus einer repräsentativen Befragung des Zentrums für Qualität in der Pflege hervorgeht (Eggert et al. 2018). Hier sahen etwa zwei Drittel der 1.000 Befragten eher Chancen und ein Viertel eher Probleme. Speziell der Einsatz von PflegeApps, Sensorik und Detektoren sowie Telepflege werden überwiegend befürwortet. Bei Pflegerobotern sank die Befürwortung mit steigender Intimität der Aufgabe - als Erinnerungshilfe für Medikamente begrüßten $76 \%$ diese Technologie, als Begleitung beim Toilettengang $51 \%$.

Vor diesem Hintergrund der breiten Diskussion des Themas Pflege und Technik lassen sich verschiedene Interessens- und Betroffenengruppen identifizieren, die jeweils eine eigene Perspektive auf das Thema einbringen: Pflegebedürftige und ihre Angehörigen, professionelle Pflegekräfte sowie politische Akteure und Kostenträger. Die nachfolgenden Ausführungen befassen sich mit der Sicht professionell Pflegender auf den Einsatz von Technik in der Pflege. Die Ergebnisse sind Teil der ersten
Befragungswelle und stellen eine Zwischenauswertung zum Thema dar.

\subsection{Forschung zu technischen Assistenzsystemen im Gesundheitsbereich}

Technische Assistenzsysteme im Gesundheitswesen können unterschiedliche Formen annehmen und verschiedene Funktionen erfüllen. In ihrem systematischen Review identifizierten Khosravi und Ghapanchi (2016) sechs Kategorien technischer Systeme (Informations- und Kommunikationstechnologien, Robotik, Telemedizin, Sensoren, Videospiele und Medikationsausgaben) zur Unterstützung von insgesamt acht klinisch relevanten Bereichen (chronische Erkrankungen, Sturzrisiko, soziale Isolation, schlechter allgemeiner Gesundheitszustand, Demenz, Unselbständigkeit, Depression und nachlässiges Medikamentenmanagement). Nach Funktionsbereichen können Pflegetechnologien zur physischen Assistenz und Mobilisierung, sozialen Begleitung und emotionalen Stütze sowie zur Kontrolle des Gesundheitszustands eingesetzt werden (Broadbent et al. 2009). Beispiele für diese Kategorien sind Fraunhofers "CareO-bot" oder die Streichelrobbe „Paro“, die vollautomatisiert ihren Aufgaben nachgehen.

Die Akzeptanz technischer Systeme wurde bei Pflegebedürftigen bereits in mehreren Studien untersucht. In Reviews mit jeweils mehr als 30 Studien wurden Faktoren wie Alter, kognitive Fähigkeiten, Bildungsstand oder bisherige Technikerfahrungen der Betroffenen, sowie Aussehen, Größe, Vertrauenswürdigkeit oder Sicherheit der Systeme identifiziert, die die Technikakzeptanz beeinflussten (Broadbent et al. 2009; Frennert und Östlund 2014; Yusif et al. 2016).

Die Akzeptanz seitens professionell Pflegender wurde hingegen bislang vergleichsweise wenig untersucht. In ihrem Review über 16 Studien zeigen Savela et al.(2017), dass Pfle- 
gende unterschiedliche Einstellungen etwa gegenüber Pflegerobotern haben. Als Ersatz für menschliche Arbeitskräfte werden sie abgelehnt und ihr Nutzen für soziale Aufgaben infrage gestellt. Hier schwingt oftmals die Angst vor dem eigenen Arbeitsplatzverlust mit. Als automatisiertes Equipment oder Transportmechanismen werden Pflegeroboter von Pflegenden jedoch positiv bewertet. Geräte zur medizinischen Kontrolle werden höher wertgeschätzt als solche zur emotionalen Unterstützung. In der ambulanten Pflege werden vor allem Telemedizin-Geräte sehr positiv bewertet, da sie bspw. Anfahrtswege von Pflegenden reduzieren konnten.

Alle Reviews sprechen sich für eine Perspektive aus, die die Sichtweisen unterschiedlicher Gruppen (Pflegende, Pflegebedürftige und ihre Angehörigen) einschließt und allen Beteiligten ein Mitspracherecht bei der Gestaltung und dem Einsatz von technischen Assistenzsystemen in der Pflege zugesteht (Bemelmans et al. 2012; Broadbent et al. 2009; Frennert und Östlund 2014). Zudem wird ein systematisches, standardisiertes Vorgehen in der Erforschung von Technikakzeptanz gefordert, um die Vergleichbarkeit von Befunden zu verbessern (Broadbent et al. 2009). Diese Aspekte werden in der vorliegenden Befragung berücksichtigt, indem die Perspektive professionell Pflegender mit validierten Instrumenten erfasst wird.

\subsection{Befragung zur Akzeptanz von Technikeinsatz in der Pflege}

\section{- - Studiendesign und Methodik}

Das Institut für Medizinische Soziologie und Rehabilitationswissenschaft der Charité Universitätsmedizin Berlin führte in Zusammenarbeit mit dem Zentrum für Qualität in der Pflege (ZQP) und unterstützt durch den Deutschen Pflegeverband (DPV) die Befragung unter professionell Pflegenden durch. Zum Einsatz kam ein Online-Fragebogen mit 130
Fragen zu allgemeinen Technikeinstellungen, Kenntnis von technischen Pflegeassistenzsystemen und der Einschätzung von Technikeinsatz in den Bereichen körperlicher, sozialer und emotionaler Unterstützung, Monitoring und Dokumentation.

Die Stichprobe der hier zugrundeliegenden ersten Befragungswelle umfasste $n=127$ Probandinnen (73\%) und Probanden (26\%) zwischen 19 und 71 Jahren. Die meisten verfügten über die mittlere Reife (45\%) oder ein Abitur (29\%) als höchste schulisch sowie eine Ausbildung (60\%) oder einen Fachhochschulabschluss (24\%) als höchste berufsbildende Abschlüsse. Die am häufigsten ausgeübten Berufe waren Krankenschwester/-pfleger (45\%) und Altenpfleger/in (22\%) in den Einrichtungen Krankenhaus (43\%), Pflegeheim (23\%) und Sozialstation (16\%). $36 \%$ der Befragten hatten eine Leitungsposition inne. Der Großteil (77 \%) verfügte über mehr als fünf Jahre Berufserfahrung.

Neben den soziodemografischen Faktoren erfasste der Fragebogen Kenntnisse über, $\mathrm{Zu}$ gang zu und Nutzung von neun beispielhaften Technologien wie Hebehilfen, Kuschelroboter (die Streichelrobbe Paro) oder Tablets zur Pflegedokumentation. Zudem gaben die Teilnehmerinnen und Teilnehmer allgemeine Selbsteinschätzungen über Technikkompetenz und -begeisterung, positive und negative Folgen von Technik an (Karrer et al. 2009). Darauf folgten Einschätzungen zum Einsatz von Technik in der Pflege allgemein und spezifisch in den vier Pflegefunktionsbereichen (1) körperliche Unterstützung, (2) soziale und emotionale Unterstützung, (3) Monitoring und (4) Dokumentation.

\section{- Ausgewählte Ergebnisse:}

Technikkenntnisse, -zugang und -nutzung - Abb. 17.1 zeigt, wie Kenntnisse über ausgewählte technische Systeme in der Pflege bei Professionellen differieren. Während der Großteil der Teilnehmerinnen und Teilnehmer Hebehilfen (99\%) und Smartphones bzw. Tablets zur Dokumentation (86\%) kennt, sind automatische Medikamentenverteiler (23\%) 


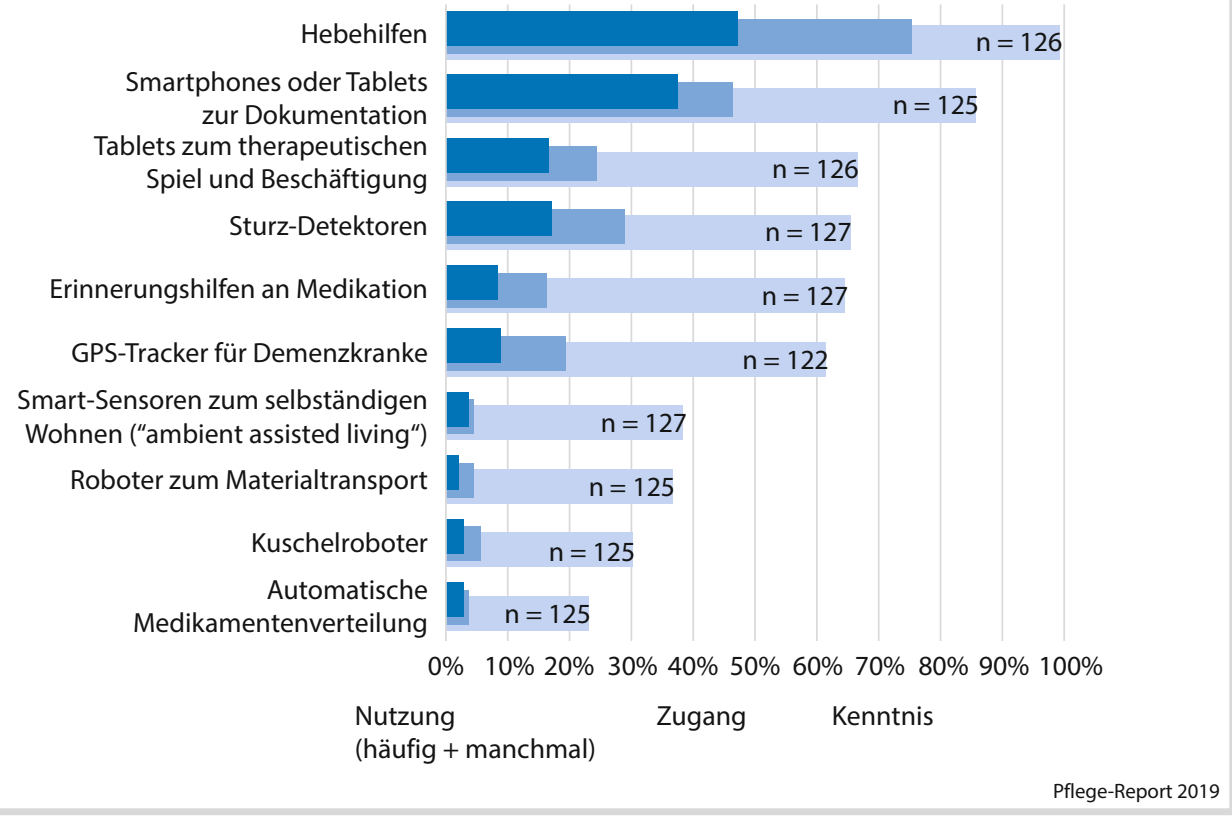

- Abb. 17.1 Kenntnisse über, Zugang zu und Nutzung von ausgewählten Assistenzsystemen

und Kuschelroboter (30\%) nur wenigen geläufig. Über alle Systeme gemittelt ist auffällig, dass trotz Kenntnis der Technik die überwiegende Mehrheit der 127 befragten Pflegekräfte (67\% im Durchschnitt) am Arbeitsplatz keinen Zugang zu ihr hat. Die Ausnahme bilden die beiden bekanntesten Systeme (Hebehilfen und Dokumentationssysteme), zu denen mehr als die Hälfte derjenigen, die sie kennen, auch einen Zugang hat. Sofern der Zugang besteht, nutzen knapp zwei Drittel der Pflegekräfte die Systeme manchmal bis häufig. Insgesamt scheint also die mangelnde Verbreitung der Technik an den Arbeitsplätzen der Pflegekräfte die entscheidende Tatsache zu sein, die den Einsatz von Technik in der pflegerischen Arbeit limitiert. Kenntnisse und Nutzung bei Bereitstellung sind über alle Systeme gesehen relativ hoch ausgeprägt.

\section{- - Ausgewählte Ergebnisse: Allgemeine Technikeinstellungen und Akzeptanz in den vier Funktionsbereichen}

Ganz allgemein zeigt sich bei den befragten Pflegekräften eine durchaus hohe Technikaf- finität - ausgedrückt durch eine hohe Technikkompetenz und Begeisterung sowie eine eher positive Einschätzung der Technikfolgen (- Abb. 17.2).

Beim Blick auf die Akzeptanz in den vier Pflegefunktionsbereichen körperliche Unterstützung, soziale und emotionale Zuwendung, Monitoring sowie Dokumentation zeigen sich zwei Tendenzen. Erstens korrelieren die Akzeptanzwerte der vier Bereiche signifikant $(.25 \leq r \leq .54)$ : Die Pflegekräfte mit niedriger Akzeptanz in einem Bereich zeigen auch niedrigere Technikakzeptanz in den anderen Bereichen. Zweitens nimmt darüber hinaus der Funktionsbereich „soziale und emotionale Unterstützung" eine Sonderrolle ein. In diesem Bereich wird der Technikeinsatz immer signifikant geringer akzeptiert als in den anderen drei Pflegefunktionsbereichen.

Dies deckt sich auch mit den Ergebnissen der Befragung zur Einstellung gegenüber Technik in der Pflege. In 12 von 13 Einstellungsfragen zeigt sich diese sehr kritische Sichtweise auf den Technikeinsatz im Bereich der emotio- 


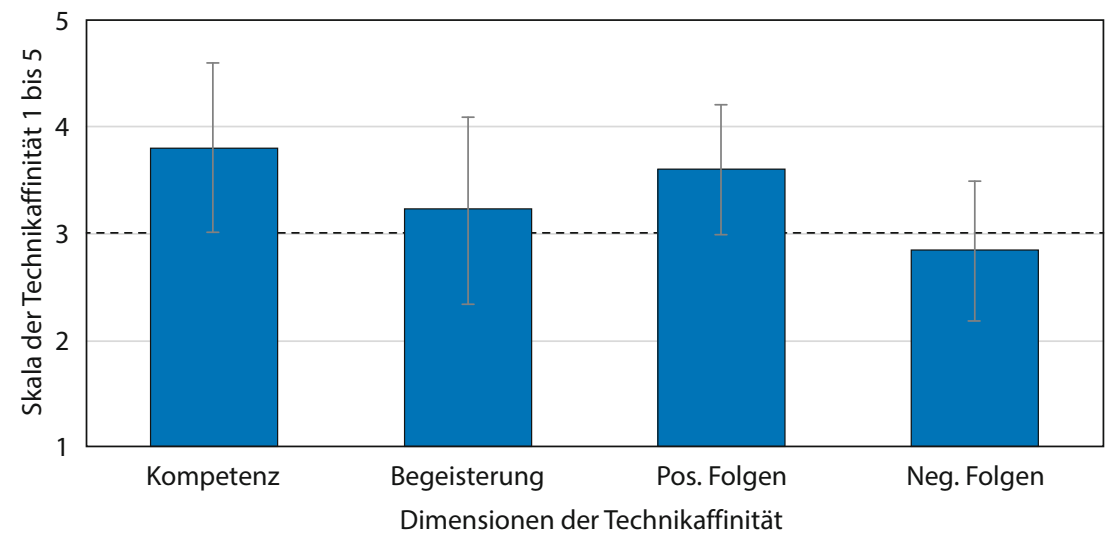

Pflege-Report 2019

- Abb. 17.2 Mittelwerte und Standardabweichungen der Technikaffinität

nalen und sozialen Unterstützung signifikant. Dieser Einsatz wird im Vergleich zu den drei anderen Funktionsbereichen als weniger hilfreich, nicht entlastend und nicht nützlich gesehen. Soziale und emotionale Zuwendung durch Technikeinsatz gefährdet nach Ansicht der 127 Befragten zudem eher den eigenen Arbeitsplatz, birgt mehr Risiken für die Gepflegten und ist stärker durch wirtschaftliche Interessen motiviert. Letztlich führe Technikeinsatz zur sozialen und emotionalen Unterstützung zum Verlust von menschlicher Wärme und stehe im Kontrast zum Berufsbild der Pflege.

- Abb. 17.3 zeigt exemplarisch die Antworten zu vier Fragen zur Technikeinstellungen und vergleicht die vier pflegerischen Funktionsbereiche körperliche Unterstützung, soziale und emotionale Unterstützung, Monitoring sowie Dokumentation.

\subsection{Fazit}

Unter den befragten 127 professionell Pflegenden zeigt sich insgesamt ein relativ hoher Kenntnisstand zu heute bereits verfügbaren technischen Assistenzsystemen. Trotz dieser Kenntnisse hat nur ein geringer Teil der Pflegekräfte breiten Zugang zu den Systemen.
Die mangelnde Verbreitung von Technik in Versorgungs- und Pflegeeinrichtungen stellt den vorliegenden Zwischenergebnissen zufolge ein wesentliches Hemmnis für die Nutzung von Technik in der Pflege dar. Wenn Technik vorhanden ist, wird sie von den meisten professionellen Pflegekräften in unserer Befragung genutzt.

Die Forschungsbefunde zeigen, dass die befragten professionellen Pflegekräfte den Einsatz von Technik zur körperlichen Unterstützung ihrer eigenen Tätigkeit zu schätzen wissen und sie als Entlastung der pflegerischen Arbeit sehen. Im Gegensatz hierzu haben sie starke Vorbehalte gegenüber Technikeinsatz zur sozialen und emotionalen Zuwendung. Damit wird deutlich, dass eine Technisierung zwischenmenschlicher Interaktionen dem professionellen Selbstverständnis der Pflegenden widerspricht. Diese Ergebnisse bestätigen damit für den deutschsprachigen Raum internationale Befunde (Alaiad und Zhou 2014; Kristoffersson et al. 2011; Savela et al. 2017).

Der Einsatz von Technik in der Pflege ist ein hochaktuelles Thema, das Potenziale und Risiken für Pflegekräfte, Pflegebedürftige und ihre Angehörigen birgt. Aus ethischer Perspektive werden bereits dringende Empfehlungen zum Umgang mit den Risiken formuliert (Bioethik- 


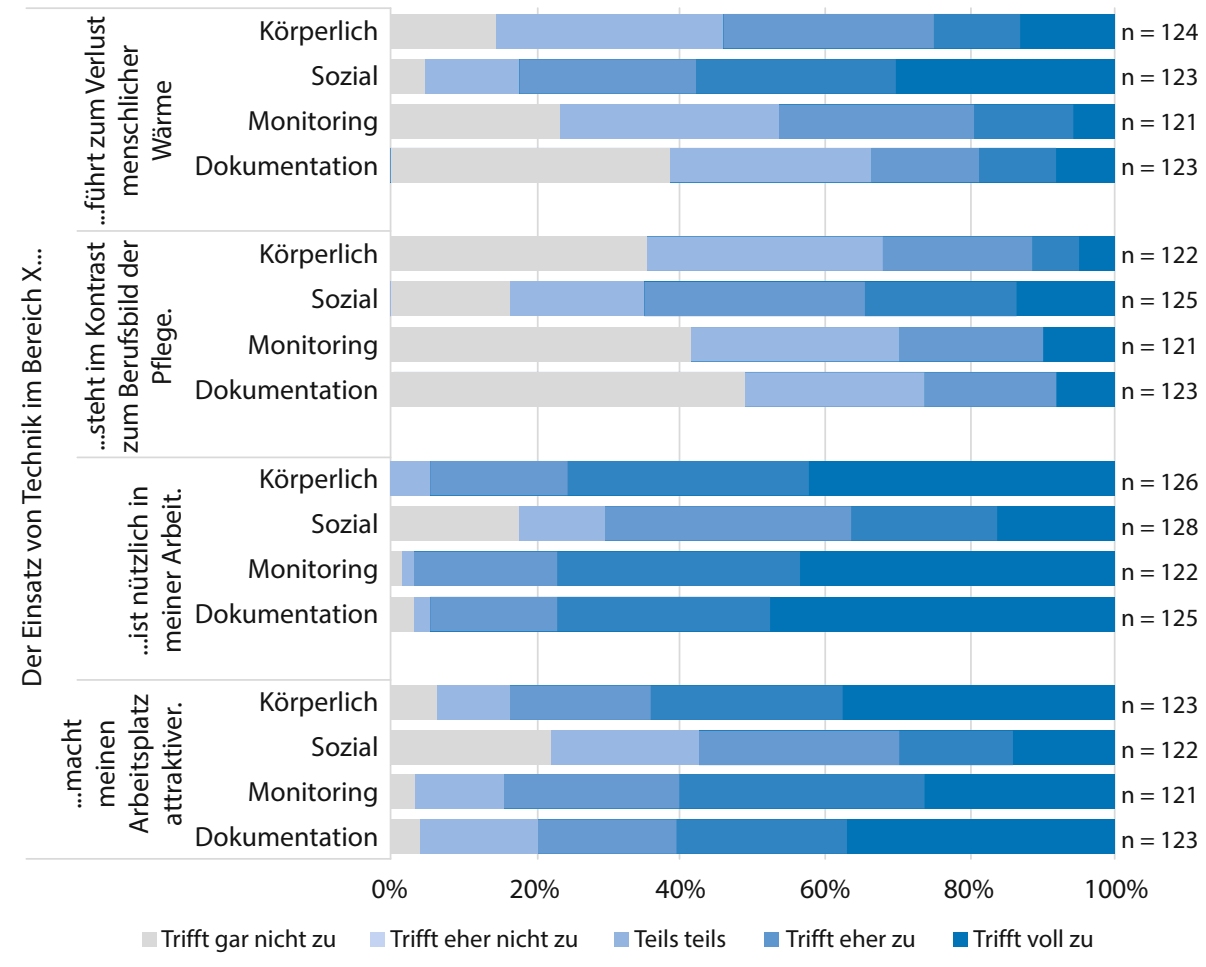

Pflege-Report 2019

- Abb. 17.3 Antworthäufigkeit zu Fragen der Technikeinstellung

kommission 2018), während die Industrie die Potenziale betont und technisierte Szenarien entwickelt (Klein et al. 2018). Die Politik ist aufgefordert, in der Gesetzgebung Handlungsund Regulierungsrahmen zu definieren.

\section{Literatur}

Alaiad A, Zhou L (2014) The determinants of home healthcare robots adoption: an empirical investigation. Med Inf (Lond) 83(11):825-840. https://doi.org/10. 1016/j.jimedinf.2014.07.003

Bemelmans R, Gelderblom GJ, Jonker P, De Witte L (2012) Socially assistive robots in elderly care: a systematic review into effects and effectiveness. J Am Med Dir Assoc 13(2):114-120

Bioethikkommission (2018) Roboter in der Betreuung alter Menschen. Bioethikkommission beim Bundeskanzleramt, Wien
BMBF (2018) Bedarfsgerechte Pflege. Menschlich und selbstbestimmt: Innovationen für eine bedarfsgerechte Pflege (https://www.technik-zummenschen-bringen.de/themen/gesundes-leben/ bedarfsgerechte-pflege)

Broadbent E, Stafford R, MacDonald B (2009) Acceptance of healthcare robots for the older population: review and future directions. Int J Soc Robot 1(4):319-330. https://doi.org/10.1007/s12369-009-0030-6

Eggert S, Sulmann D, Teubner C (2018) Einstellung der Bevölkerung zu digitaler Unterstützung in der Pflege. Zentrum für Qualität in der Pflege (ZQP), Berlin

Fachinger U (2017) Technikeinsatz bei Pflegebedürftigkeit. In: Jacobs K, Kuhlmey A, Greß S, Klauber J, Schwinger A (Hrsg) Pflege-Report 2017. Schwerpunkt: Die Versorgung von Pflegebedürftigen. Schattauer, Stuttgart

Frennert S, Östlund B (2014) Review: seven matters of concern of social robots and older people. Int J Soc Robot 6(2):299-310. https://doi.org/10.1007/ s12369-013-0225-8 
Karrer K, Glaser C, Clemens C, Bruder C (2009) Technikaffinität erfassen-der Fragebogen TA-EG. Mensch Im Mittelpkt Tech Syst 8:196-201

Khosravi P, Ghapanchi AH (2016) Investigating the effectiveness of technologies applied to assist seniors: a systematic literature review. Med Inf (Lond) 85(1):1726. https://doi.org/10.1016/j.ijmedinf.2015.05.014

Klein B, Graf B, Schlömer IF, Roßberg H, Röhricht K, Baumgarten S (2018) Robotik in der Gesundheitswirtschaft. Einsatzfelder und Potentiale. medhochzwei, Heidelberg

Kristoffersson A, Coradeschi S, Loutfi A, SeverinsonEklundh K (2011) An exploratory study of health professionals' attitudes about robotic telepresence technology. J Technol Hum Serv 29(4):263-283. https://doi.org/10.1080/15228835.2011.639509
Rossmann ED, Albani S, Röspel R, Espendiller M, Brandenburg M, Lenkert R, Christmann A (2018) Robotik und assistive Neurotechnologien in der Pflege gesellschaftliche Herausforderungen. Ausschuss für Bildung, Forschung und Technikfolgenabschätzung, Berlin

Savela N, Turja T, Oksanen A (2017) Social acceptance of robots in different occupational fields: a systematic literature review. Int J Soc Robot 10(4):493-502. https://doi.org/10.1007/s12369-017-0452-5

Yusif S, Soar J, Hafeez-Baig A (2016) Older people, assistive technologies, and the barriers to adoption: a systematic review. Med Inf (Lond) 94:112-116

Open Access Dieses Kapitel wird unter der Creative Commons Namensnennung 4.0 International Lizenz (http:// creativecommons.org/licenses/by/4.0/deed.de) veröffentlicht, welche die Nutzung, Vervielfältigung, Bearbeitung, Verbreitung und Wiedergabe in jeglichem Medium und Format erlaubt, sofern Sie den/die ursprünglichen Autor(en) und die Quelle ordnungsgemäß nennen, einen Link zur Creative Commons Lizenz beifügen und angeben, ob Änderungen vorgenommen wurden.

Die in diesem Kapitel enthaltenen Bilder und sonstiges Drittmaterial unterliegen ebenfalls der genannten Creative Commons Lizenz, sofern sich aus der Abbildungslegende nichts anderes ergibt. Sofern das betreffende Material nicht unter der genannten Creative Commons Lizenz steht und die betreffende Handlung nicht nach gesetzlichen Vorschriften erlaubt ist, ist für die oben aufgeführten Weiterverwendungen des Materials die Einwilligung des jeweiligen Rechteinhabers einzuholen.

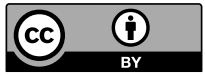

